\title{
Photopolarization Effect and Photoelectric Phenomena in Layered GaAs Semiconductors *
}

\author{
Yuo-Hsien Shiau \\ Graduate Institute of Applied Physics, National Chengchi University \\ Taiwan, Republic of China
}

\section{Introduction}

The studies of transport properties in semiconductors have made great progresses for the past decades. This is mainly due to the advanced technologies for development of new materials and the application of nonlinear dynamics to the fundamental well-known materials. In particular, the discipline of nonlinear dynamics grows fast, which is due to the cooperation of theoretical background and experimental findings. Among systems considered, semiconductors represent interesting and highly productive examples of the experimental investigation of nonlinear dynamics. One of the typical findings observed in nonlinear semiconductors is the dynamics of propagating electrical solitary waves which could be periodic or chaotic. Many of these phenomena have been studied in bulk semiconductors as well as superlattices, and can be successfully explained by means of theoretical as well as numerical approaches (Amann \& Schöll, 2005; Bonilla \& Grahn, 2005; Cantalapiedra et al., 2001; Gaa \& Schöll, 1996; Wack, 2002). Of particular interest is that GaAs semiconductors have been shown to generate microwave radiation. The generation was attributed to propagating space-charge waves (or high-field domains). The domain shape parameters such as the maximum fields and the domain size are controllable with changing the concentration of ionized donors that are doped in the semiconductor substrate.

It is known that nonlinear electro-optic characteristics can be observed in an $n^{+}-n^{-}-n-n^{+}$ GaAs sandwich structure under optical excitation, where potential applications including optical control of microwave output, ultrafast electric switches, memory cells and other areas. The key factor in such a system is that propagating space-charge waves (SCWs) were formed at the cathode and destroyed at (or before) the anode being due to a balance of the diffusion of carriers and the nonlinearity in the velocity-field characteristic, where it can be realized that propagating SCWs are equivalent to the case of the laser beam propagation in Kerr-type nonlinear optical media. Besides, the notch profile (i.e., the $\mathrm{n}^{-}$layer) will be strongly influenced by the optical illumination, which will result in the tuning traveling-distance of SCWs. Owing to that, optical control of microwave output can be expected, and this phenomenon is related to the photopolarization effect. In addition, the interesting phenomena including optically induced hysteresis and long-lived transient behaviors can be observed in a layered semiconductor. In the meanwhile, the development of multiple sandwich structures has been known to be helpful for the high-power microwave generation; however, electro-optic characteristics are less known in this system. Concerning on multiple sandwich

*This work was partially supported by the National Science Council of the Republic of China (Taiwan) under Contract Nos. NSC 98-2112-M-004-001-MY3 
structures without laser illumination, it would be expected that coherent/identical SCWs initiated from different doping notches will show up, but it has never been reported that persistent photoelectric phenomena can be observed in multiple GaAs sandwich structures due to the photopolarization effect. In this Chapter, using 10-ns-duration pulse of a Nd:YAG laser to generate electron-hole pairs is considered. Interestingly, both exponential and non-exponential photoelectric relaxations can be numerically observed via the well-accepted drift-diffusion model; therefore, the switching time for non-exponential relaxations would be much higher than that of exponential relaxations. Thus, photo-induced persistent charge transport can be discovered in the present multiple GaAs sandwich structures, which is believed to be important properties of opto-electronic and transport processes in layered semiconductors.

The remainder of this Chapter is organized as follows. Optically induced hysteresis, the photopolarization effect, and non-exponential photoelectric relaxations will be introduced in Sec. 2. Sec. 3 will provide a numerical evidence of long-lived transient behaviors under the consideration of optical stochasticity. Concluding remarks will be given at the end of this Chapter.

\section{Photoelectric phenomena}

The physical basis for hysteretic switching between low- and high- conducting states in nonlinear semiconductors is usually related to the exhibition of S-shaped negative differential conductivity (SNDC) on the current-density-field characteristic. Up to now, several mechanisms have been proposed to induce SNDC. For example, two-impurity-level model with impact ionization (II) for $n$-GaAs at $4.2 \mathrm{~K}$ (Schöll, 1987), the interband breakdown for $n$-GaAs at room temperature (Gel'mont \& Shur, 1970), and generic N-shaped NDC characteristics connected with a large load resistance (LR) (Döttling \& Schöll, 1992; Shiau \& Cheng, 1996), i.e., inverted SNDC, etc. Theoretical analyses and predictions, under the assumption of spatial homogeneity, are usually based on the local or global bifurcation schemes around the operating points. However, in whatever theoretical models II or LR is a key factor to induce SNDC. In this section we numerically demonstrate, even without consideration of II and LR, the hysteretic switching in an $n^{+}-n^{-}-n-n^{+}$GaAs sandwich structure (Oshio \& Yahata, 1995) under local optical excitation. It is interesting to find that quenched and transit modes can coexist at the same laser intensity. And the transition between these two dynamical states is hysteretic, i.e., optically induced hysteresis. These results also indicate using a layered semiconductor as an inverter of optical input to microwave output and this electro-optic phenomenon shall be potentially useful for applications. Moreover, in realistic situations the electric field in SCWs could become strong enough to generate electron-hole pairs due to the II effect. Therefore, considering the influence of the II effect on this hysteresis is also performed. The numerical results show that this hysteretic switching still can be maintained but the transition regions between these two dynamical states will be perturbed. Thus, the II effect is not a primary factor in our system. This is why we called optically induced hysteresis, a novel nonlinear electro-optic characteristic, between two different conducting states in a semiconductor device.

Before going to introduce our computational model, it shall be noted that the function of this doping notch is to establish local space-charge field for dipole-domain nucleation. In order to study the bipolar transport, we further consider local optical excitation which is close to the doping notch. We expect the domain dynamics originally determined by the doping notch and external dc bias will be influenced by the optical intensity. The motivation of consideration of local optical excitation in the active region is to redistribute the space-charge 
field around the doping notch via optical generation of hole carriers. More precisely speaking, local optical excitation is to make doping notch as a collector of electrons. Then the internal field in doping notch will become stronger, which can speed up electrons and influences the dipole-domain nucleation. Therefore, if the optical intensity is large enough, even at lower dc bias, the quenched domain could be controlled and possibly becomes the transit domain. Thus the so-called optical control of electrical propagations in a GaAs-based Gunn device is expected.

As the sandwich structure we consider a GaAs-based semiconductor device with the doping profile $N_{D}(x)$, denoted as $\mathrm{n}^{+}(8.0 \mu \mathrm{m})-\mathrm{n}^{-}(4.0 \mu \mathrm{m})-\mathrm{n}(40.0 \mu \mathrm{m})-\mathrm{n}^{+}(8.0 \mu \mathrm{m})$, shown in Fig. 1 . The active region is sandwiched between the highly-doped $\mathrm{n}^{+}$cathode and anode regions. A $4 \mu \mathrm{m}$ doping notch, at the beginning of the active region, is to initiate the dipole domain near the cathode. In addition, the laser illumination with $3 \mu \mathrm{m}$ width is considered and denoted as $I(x)$. The detailed model equations and the GaAs parameters used for numerical simulation are listed in Tables I and II, respectively (Shiau \& Peng, 2005). In the following the results obtained from direct numerical simulation with the consideration of the drift-diffusion model and fixed boundary conditions will be demonstrated in detail.

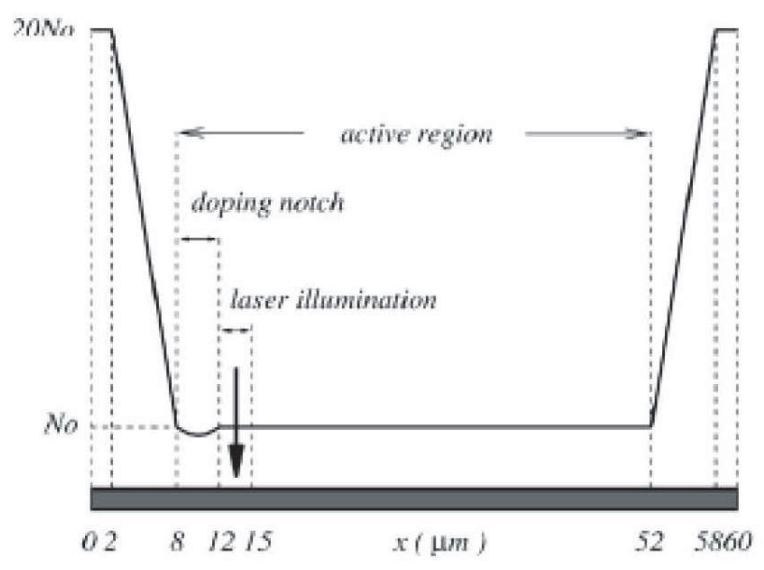

Fig. 1. Schematic illustration of device doping profile $N_{D}(x)$ and local laser illumination $I(x)$. The sample length is $60 \mu \mathrm{m}$.

Without laser illumination and II effect, the simulated model shall display traditional quenched or transit domains which are dependent of the external dc bias (Sze, 1969). When external bias is $12 \mathrm{~V}$, the dynamical characteristics of the quenched mode are clearly exhibited via spatiotemporal behaviors of the electric field and time-dependant total current density in Fig. 2. A high-field domain (Fig. 2(a)) grows from the doping notch then annihilate before reaching the anode. The waveform of oscillating current density $J$ is shown in Fig. 2(b) and the associated oscillating frequency $f_{0}$ is around $20 \mathrm{GHz}$. Of course, in this case the hole density shall be zero in the whole sample. If external bias is increased to $20 \mathrm{~V}$, the transit mode, also clearly shown in Fig. 2, cyclical propagation from the cathode to the anode is obtained. And the oscillating current frequency is around $2 \mathrm{GHz}$. As the case of the quenched domain, $p$ is still zero in the whole computational domain. Therefore, the simulated model is well to describe the traditional electrical-induced domain formation and propagation. Now we consider local laser illumination $I(x)$ applied to the semiconductor device which is operated 


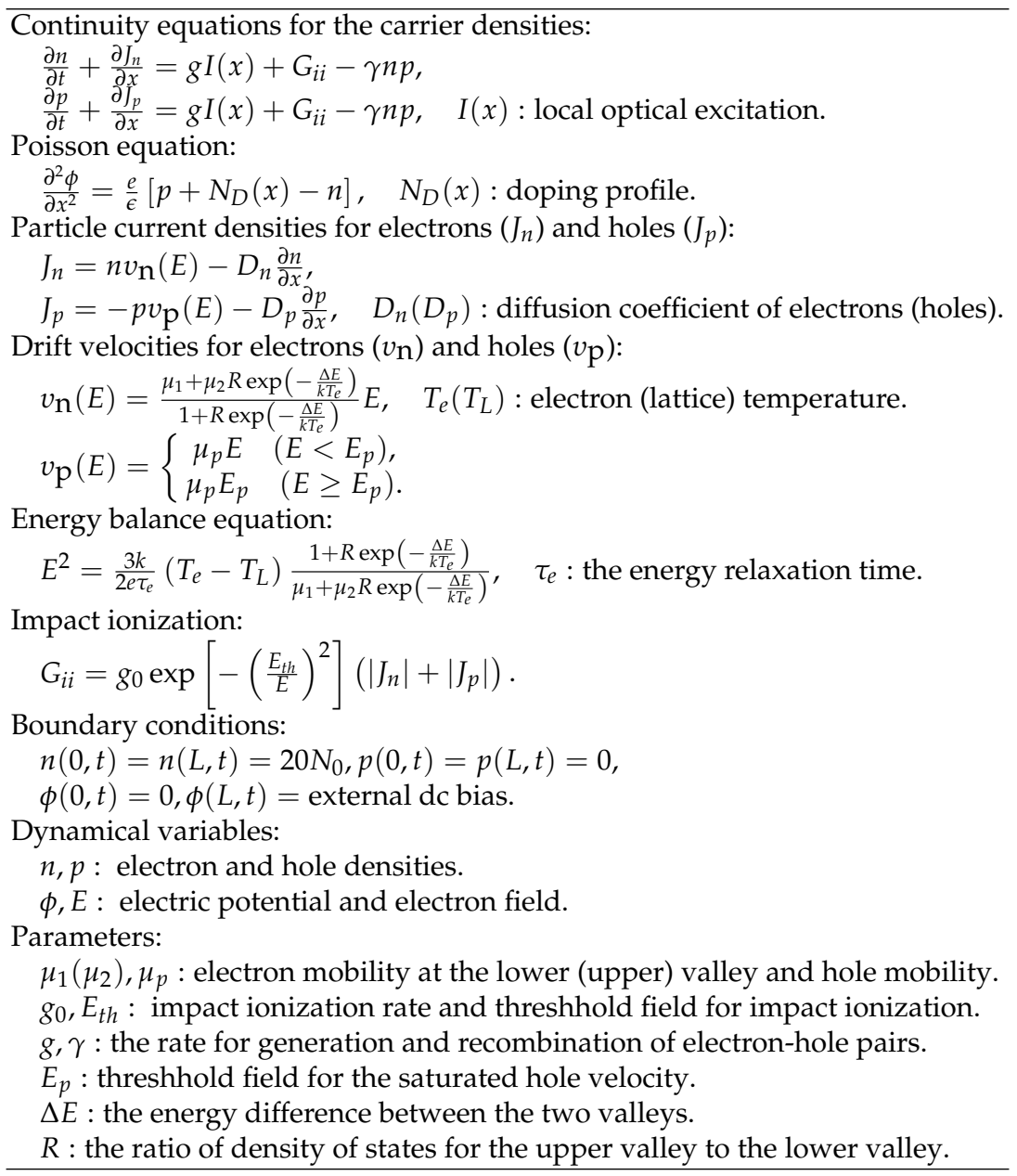

Table 1. Model equations.

at $12 \mathrm{~V}$. When the applied laser intensity is $192 \mathrm{~kW} / \mathrm{cm}^{2}$, a transit domain with a smaller domain width (compared to the upper portion of Fig. 2(a)) is shown in Fig. 3. Not surprising, the stationary and nonuniform hole distribution is obtained around the notch region (Fig. 3). The oscillating current frequency in this case is also around $2 \mathrm{GHz}$. As we already mentioned that the optical generation of hole carriers is to control the space-charge field of the doping notch. When the suitable laser intensity is applied, the transition from the quenched mode to the transit mode at lower dc bias still can be observed. Therefore, the optical control of electrical propagations is numerically demonstrated. Besides, these two different electrical propagations can coexist at the same laser intensity. Fig. 4 illustrates the formation of quenched and transit modes when the laser intensity is $96 \mathrm{~kW} / \mathrm{cm}^{2}$. Thus this kind of bistable characteristic is due to the optical excitation, which is little known in $n^{+}-n^{-}-n-n^{+}$ 


\begin{tabular}{cccc}
\hline \hline parameter & value & parameter & value \\
\hline$D_{n}$ & $200 \mathrm{~cm}^{2} / \mathrm{s}$ & $R$ & 94 \\
$D_{p}$ & $20 \mathrm{~cm}^{2} / \mathrm{s}$ & $k$ & $8.6186 \times 10^{-5} \mathrm{eV} / \mathrm{K}$ \\
$\epsilon$ & $1.17 \times 10^{-12} \mathrm{~F} / \mathrm{cm}$ & $\Delta E$ & $0.31 \mathrm{eV}$ \\
$e$ & $1.61 \times 10^{-19} \mathrm{C}$ & $T_{L}$ & $300 \mathrm{~K}$ \\
$g$ & $4.4 \times 10^{18} \mathrm{~cm}^{-1} \mathrm{sec}^{-1} \mathrm{~W}^{-1}$ & $\tau_{e}$ & $10^{-12} \mathrm{~s}$ \\
$\gamma$ & $10^{-10} \mathrm{sec}^{-1} \mathrm{~cm}^{3}$ & $N_{0}$ & $5 \times 10^{15} \mathrm{~cm}^{-3}$ \\
$\mu_{1}$ & $8500 \mathrm{~cm}^{2} / \mathrm{V} \cdot \mathrm{s}$ & $g_{0}$ & $2 \times 10^{5} \mathrm{~cm}^{-1}$ \\
$\mu_{2}$ & $50 \mathrm{~cm}^{2} / \mathrm{V} \cdot \mathrm{s}$ & $E_{t h}$ & $550 \mathrm{kV} / \mathrm{cm}$ \\
$\mu_{p}$ & $400 \mathrm{~cm}^{2} / \mathrm{V} \cdot \mathrm{s}$ & $E_{p}$ & $40 \mathrm{kV} / \mathrm{cm}$ \\
\hline
\end{tabular}

Table 2. The fundamental constants and GaAs parameters for numerical simulation.

semiconductor devices. We carefully check the transition in between quenched and transit modes, and find that this transition is hysteretic. The transition regions corresponding to the quenched mode to the transit mode and vice versa are observed at $132 \mathrm{~kW} / \mathrm{cm}^{2}$ and 82 $\mathrm{kW} / \mathrm{cm}^{2}$, respectively. The detailed electro-optic characteristic of this two different electrical propagations is illustrated in the upper portion of Fig. 5 via oscillating current frequency $f$ versus laser intensity I plot. The circular and triangular symbols denote, respectively, quenched and transit domains. It is clear to see that the oscillating current frequency of the quenched domain gradually decreases when $I$ increases. Moreover, the oscillating current frequency of the transit domain is independent of the laser intensity, which means that the traveling time of the transit domain is only related with the bulk property and not influenced by the local optical excitation. In addition, the inset in the upper portion of Fig. 5 is the corresponding hysteretic $J_{\min }-I$ curve when $I$ is slowly increased (top) and decreased (bottom), where $J_{\min }$ is the extreme minimum current density. Moreover, the calculated electric field of quenched and transit domains is much small than the $E_{t h}(=550 \mathrm{kV} / \mathrm{cm})$ (Hall \& Leck, 1968), i.e., at least less than one order of magnitude. Therefore, the II process to generate electron-hole pairs in high-field domains can be neglected. Nevertheless, in the following we still investigate the influence of the II effect on the hysteretic $f$-I and $J_{\min ^{-}}-I$ plots. The lower portion of Fig. 5 clearly shows that the influence of the II effect is only to perturb the transition regions which become at $122 \mathrm{~kW} / \mathrm{cm}^{2}$ and $77 \mathrm{~kW} / \mathrm{cm}^{2}$. Compared to the upper portion the threshold laser intensities for domain transition are all decreased. The reasonable explanation is that in addition to the local optical excitation the internal field in doping notch will become stronger due to the generation of hole carriers via the nonlinear amplification of the II effect. Therefore, the laser intensity needed for domain transition will decrease. The inset in the lower portion of Fig. 5 is also the corresponding hysteretic $J_{\min }-I$ curve.

Here, we would like to give a more detailed explanation for Fig. 5. It is well known that the notch profile affects strongly on the shedding of the high-field domain. In this study the doping notch illustrated in Fig. 1 can be changed due to the local optical excitation. This result we called the photopolarization effect. At low illumination, a stationary hole profile is established nearby the $\mathrm{n}^{-}$layer. Therefore, the original dipole field owing to the $\mathrm{n}^{-}$layer will be enhanced by the present hole distribution. In other words, the length and associated profile of the $\mathrm{n}^{-}$layer are effectively as well as perturbatively changed. This phenomenon finally will result in the tuning traveling-distance of the quenched domain. At high illumination, the hole distribution can extend to the cathode emitter (i.e., the $\mathrm{n}^{+}$layer) and leads to a significant enhancement of the dipole field. Consequently, the effective length and associated profile of the $\mathrm{n}^{-}$layer have a dramatic change, which result in the transit dynamics even at a lower $\mathrm{dc}$ 

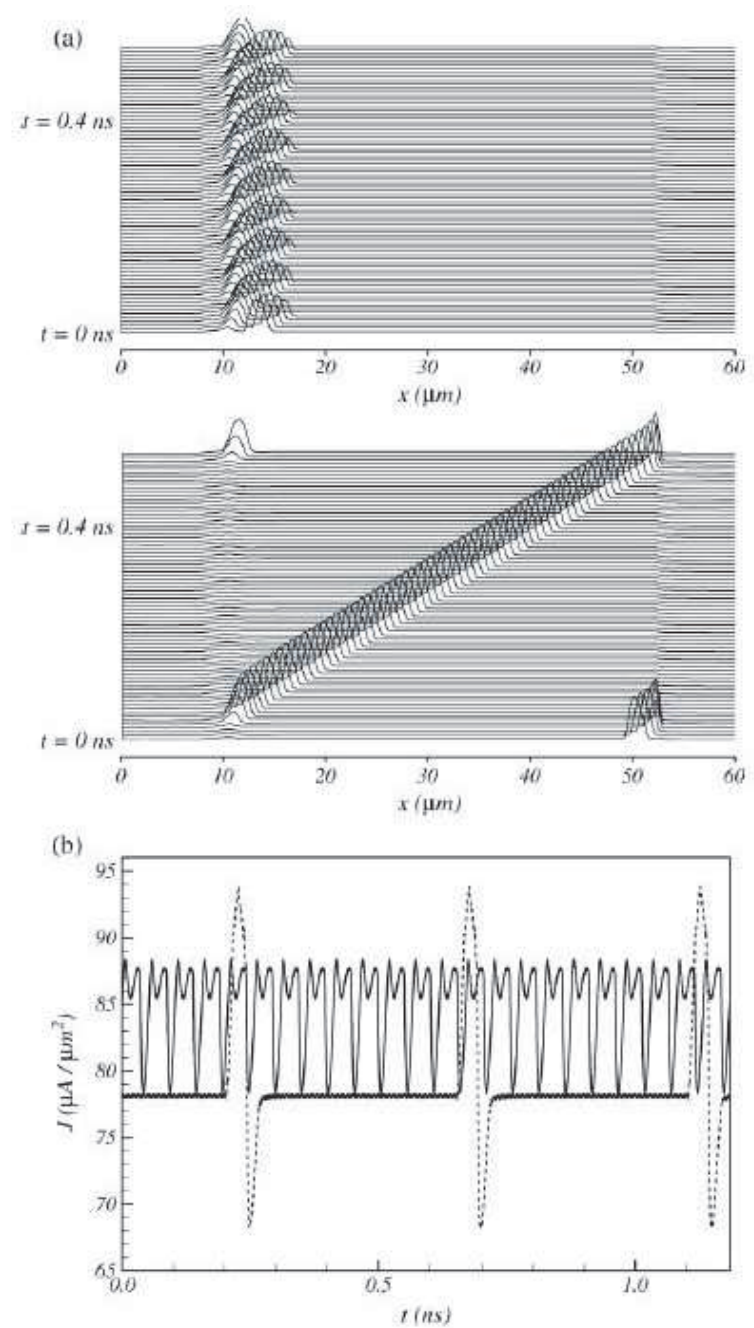

Fig. 2. Without consideration of local laser illumination and the II effect, the dynamical characteristics of the quenched and transit modes for dc bias, respectively, being equal to 12 $\mathrm{V}$ and $20 \mathrm{~V}$. (a) the quenched domain in upper portion and the transit domain in lower portion. (b) the time evolution plot of the total current density for the transit mode (dashed line) and the quenched mode (solid line). The maximum values of electric fields in (a) are $84.0 \mathrm{kV} / \mathrm{cm}$ for the transit domain and $35.5 \mathrm{kV} / \mathrm{cm}$ for the quenched domain.

bias. In addition to the mention above, it is also interesting to find that there is a hysteretic transition of the hole distribution when laser illumination is varied from low to high and vice versa. Therefore, the nonlinear electro-optic characteristic in Fig. 5 can be explained as a result of the photopolarization effect. The detailed quantitative analysis of the effective notch profile 

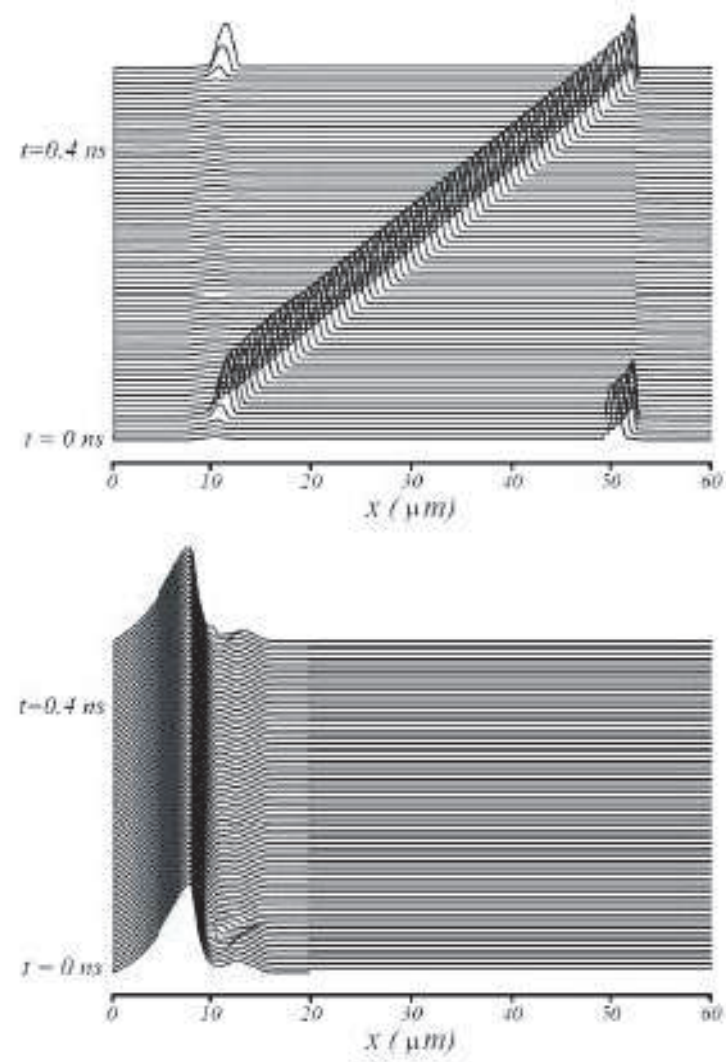

Fig. 3. Illustrations of dynamical characteristics for the transit mode when this semiconductor device is operated at $12 \mathrm{~V}$ and illuminated with $192 \mathrm{~kW} / \mathrm{cm}^{2}$. The spatiotemporal behaviors of electric fields and hole densities are shown in, respectively, upper portion and lower portion. The maximum values of electric fields and hole densities are, respectively, equal to $49.7 \mathrm{kV} / \mathrm{cm}$ and $2.77 \mathrm{a} \tilde{N} 1015 \mathrm{~cm}^{-3}$.

influenced by the lager illumination was reported via the cross-correlation matrix method (Shiau, 2006).

Concerning on multiple sandwich structures, our system is a series combination of two identical $\mathrm{n}^{+}(4.0 \mu \mathrm{m})-\mathrm{n}^{-}(2.0 \mu \mathrm{m})-\mathrm{n}(20.0 \mu \mathrm{m})-\mathrm{n}^{+}(4.0 \mu \mathrm{m})$ layered semiconductors, which is biased at $12 \mathrm{~V}$. Without the consideration of optical illumination, coherent/identical SCWs initiated from different doping notches will show up. The drift velocity, traveling distance, and cyclic period for each of SCWs would be approxmatively equal to $0.8 \times 10^{7} \mathrm{~cm} / \mathrm{s}, 2 \mu \mathrm{m}$, and $0.025 \mathrm{~ns}$, respectively. In addition, the potential drop across the first (or second) layered semiconductor is $6 \mathrm{~V}$. However, when the locally illuminated $\mathrm{n}$ region adjacent to the doping notch (i.e., $1.5 \mu \mathrm{m}$ illumination) is considered, it would be interesting to find non-identical SCWs in these two layered semiconductors. Fig. 6 depicts that the potential drop $\phi$ across the second layered semiconductor is a function of time, where 10-ns-duration pulse of a $\mathrm{Nd}$ :YAG laser is switched on at $t=0$, and the dashed (solid) line is resulted from laser 


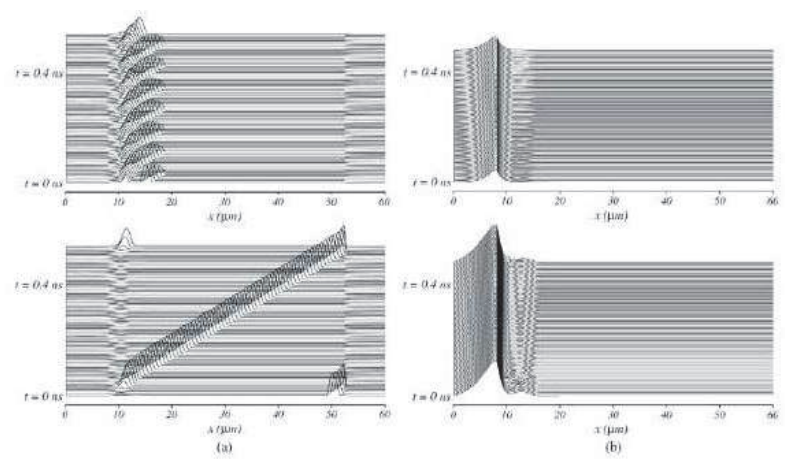

Fig. 4. Coexistence of different electrical propagations when the laser intensity is decreased to $96 \mathrm{~kW} / \mathrm{cm}^{2}$ and the dc bias is still kept at $12 \mathrm{~V}$. (a) the quenched domain in upper portion and the transit domain in lower portion. (b) upper portion: hole distribution for the quenched mode and lower portion: hole distribution for the transit mode. The maximum values of electric fields in (a) are $49.7 \mathrm{kV} / \mathrm{cm}$ for the transit domain and $36.8 \mathrm{kV} / \mathrm{cm}$ for the quenched domain. As for the maximum values of hole densities in (b) are $2.77 \mathrm{a} \tilde{N} 10^{1} 5 \mathrm{~cm}^{-3}$ for the transit mode and $0.98 \mathrm{a} \tilde{N} 10^{1} 5 \mathrm{~cm}^{-3}$ for the quenched mode.

illumination on the second (first) layered semiconductor. During $10 \mathrm{~ns}$ illumination, $\phi$ exhibits complicated dynamical response including exponential decrease (or exponential increase) as well as oscillating patterns, where the oscillating period is roughly equal to $0.045 \mathrm{~ns}$ (see the inset shown in Fig. 6). When the illumination is switched off at $t=10 \mathrm{~ns}$, exponential and non-exponential photoelectric relaxations will show up.

The exponential decrease (increase) during $10 \mathrm{~ns}$ illumination reflects the discharge (charge) process of the second layered semiconductor, and the oscillating pattern is associated with the formation of new SCWs. Moreover, non-identical SCWs in these two layered semiconductors can be expected. After $10 \mathrm{~ns}$ illumination, the exponential (non-exponential) photoelectric relaxation represents a recharge (discharge) process. In particular, the discharge process displays a non-monotonic behavior, where the potential drop $\phi$ quickly crosses the equilibrium value $6 \mathrm{~V}$ and slowly goes back to $6 \mathrm{~V}$. Thus, photo-induced persistent charge transport can be discovered in the present multiple GaAs sandwich structures

Finally, we would like to discuss the validity of the present approach. The simulated model is a combination of the well-known drift diffusion equations, a single electron temperature model, and fixed boundary conditions. To our knowledge, it can successfully describe the domain dynamics for longer devices (Shaw et al., 1992). The parameters used in this simulation for GaAs are taken from Refs.(Yeh, 1993; Sze, 1969), while the coefficients of impact ionization are from Ref.(Hall \& Leck, 1968). Another important issue we would like to discuss is the laser properties. the interesting phenomena in this study occur at laser intensity with the order of magnitude from $10^{4} \mathrm{~W} / \mathrm{cm}^{2}$ to $10^{5} \mathrm{~W} / \mathrm{cm}^{2}$. As far as we know, such conditions are experimentally feasible since two wave mixing experiments in GaAs have been performed with light at more than $10 \mathrm{MW} / \mathrm{cm}^{2}$ intensity (Disdier \& Roosen, 1992; Duba et al., 1989). And the laser source we considered is 10-ns-duration pulse of a Nd:YAG laser with wavelength $1064 \mathrm{~nm}$, which ensures our simulation results can be observed in this pulse laser. As for the technique of $3 \mu \mathrm{m}$ (or $1.5 \mu \mathrm{m}$ ) laser illumination also can be easily performed via GRIN (Chen, 1996), i.e., graded refractive index lenses. Therefore, we believe that our numerical results could be observed experimentally. 

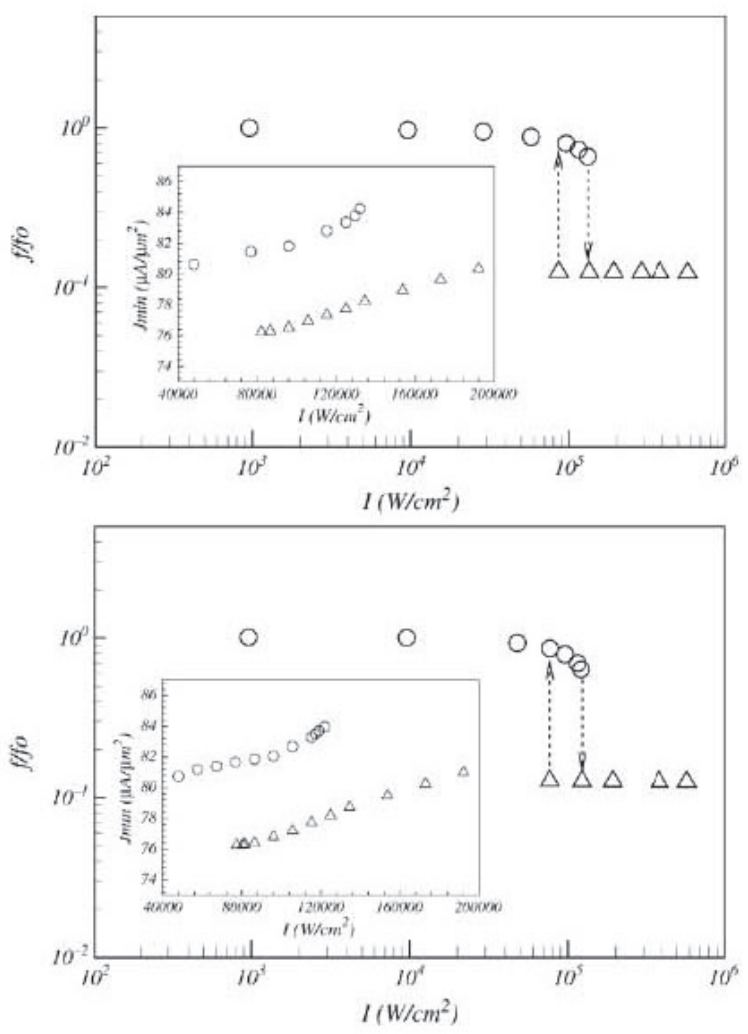

Fig. 5. The upper portion: the nonlinear electro-optic characteristic when exclusion of the II effect. The corresponding $J_{\min }-I$ hysteretic curve is shown in the inset. The lower portion: the same illustrations as in the upper portion but inclusion of the II effect.

\section{Long-lived transient behaviors with optical stochasticity}

In this section, we report the electrical response with long-lived transient characteristic, i.e., lock-on effect, in the above-mentioned GaAs-based Gunn device under stochastic stimuli. Our results can be considered as a numerical evidence for the new development of nonlinear theory which describes the so-called noise delayed decay (NDD) of unstable dynamical states (Agudov \& Malakhov, 1999; Horsthemke \& Lefever, 1984). Furthermore, this nonequilibrium decay process in our system can be used to control the response time of an ultrafast microwave switch (i.e., a nanosecond switch). To the best of our knowledge, the nonequilibrium decay rate in realistic semiconductors influenced by the optical stochasticity has never been reported. Moreover, the concept of NDD can be generally applied to other semiconductor systems with unstable dynamical states. For example, semiconductors with persistent photoconductivity can be used as many important applications. However the locking time of the dark current (i.e., an unstable dynamical state) is fundamentally related with material preparation (Joshi et al., 1999). According to the NDD effect, the locking time can possibly be prolonged and 


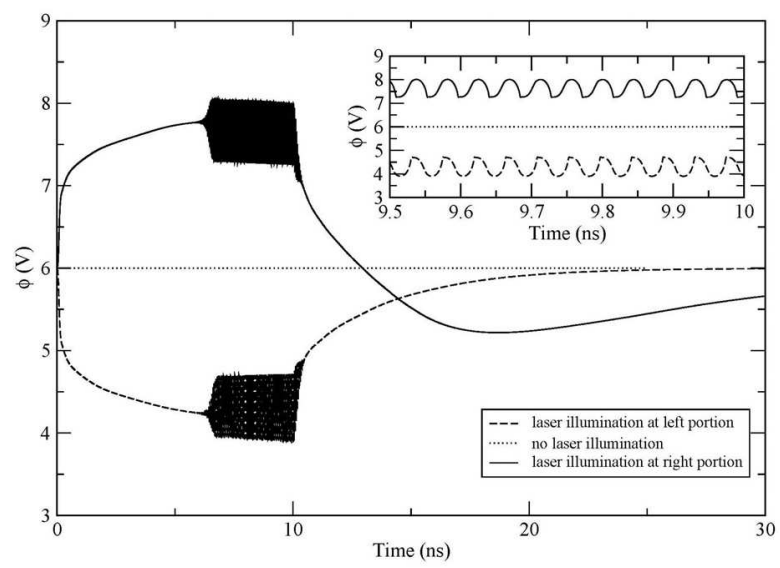

Fig. 6. Exponential and non-exponential photoelectric relaxations in multiple sandwich structures.

controlled. Therefore, the NDD effect developed by the nonlinear theory may play a crucial role for the semiconductor industry.

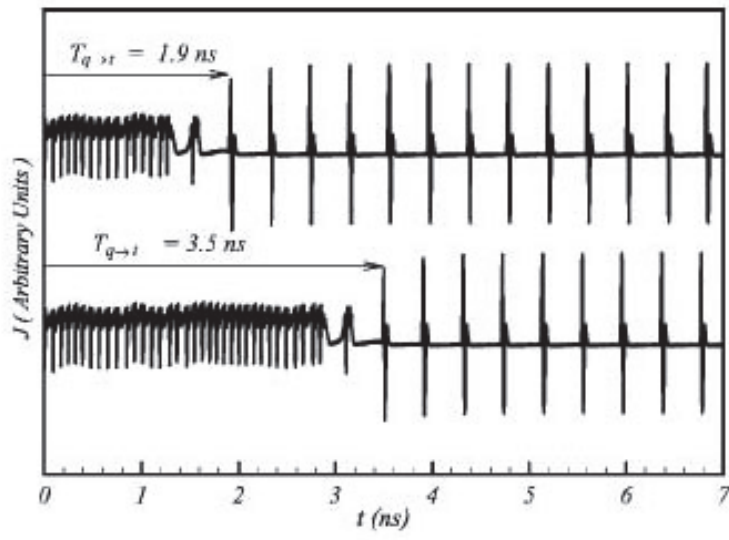

Fig. 7. Time evolution plot of the transient $J$ values for laser intensity beginning at 115 $\mathrm{kW} / \mathrm{cm}^{2}$ and then suddenly increased to $125 \mathrm{~kW} / \mathrm{cm}^{2}$. The upper portion: without the additional randomness, the switching time $T_{q \rightarrow t}$ is $1.9 \mathrm{~ns}$. The lower portion: when $A$ is $10^{-2}$, $T_{q \rightarrow t}$ is increased to $3.5 \mathrm{~ns}$.

The upper portion of Fig. 7 illustrates the transient current oscillation from the optically-induced quenched domain to transit domain with the laser intensity initially at 115 $\mathrm{kW} / \mathrm{cm}^{2}$ then increased suddenly to $125 \mathrm{~kW} / \mathrm{cm}^{2}$. The time location of the first extreme maximum current density for optically-induced transit domain is defined as the switching time $T_{q \rightarrow t}$ from the optically-induced quenched to transit domain. The switching time in this case is around $1.9 \mathrm{~ns}$. This is why a GaAs-based Gunn device can be regarded as an ultrafast microwave switch. If we further consider the additional weak noise in the laser 
intensity, the local uniform laser illumination $I(x)$ shall be replaced by $I(x)[1+A \eta(t)]$ with an additional requirement $1+A \eta(t) \geq 0$. Here $A$ is the strength of the noise, and $\eta(t)$ must satisfy the white noise conditions: $\langle\eta(t)\rangle=0$ and $\left\langle\eta(t) \eta\left(t^{\prime}\right)\right\rangle=\delta\left(t-t^{\prime}\right)$. The symbol \langle\rangle denotes the ensemble average. The lower portion of Fig. 7 demonstrates that the switching time is increased to $3.5 \mathrm{~ns}$ with $A=10^{-2}$. It shall be noted that the additional randomness is just given at laser intensity being equal to $125 \mathrm{~kW} / \mathrm{cm}^{2}$ for the artificial control of electrical response. The corresponding spatiotemporal dynamics of Fig. 7 are shown in Fig. 8 via contour plots. The white area represents the higher electric-field values and intermediate shading from gray to black shows different levels of lower electric-field values. It is seen clearly that the optically-induced quenched domain temporally locks in the space, which is strongly dependent of the additional randomness. The detailed relation between $T_{q \rightarrow t}$ and $A$ is plotted with squared symbols in Fig. 9. It is found that not only the prolonged switching time but also the nonlinear $T_{q \rightarrow t}(A)$ spectrum. When $A$ exceeds $10^{-2}$, the switching time is fixed at $3.5 \mathrm{~ns}$, while the NDD effect disappears as $A \leq 10^{-3}$. Now we discuss the switching time $T_{t \rightarrow q}$ from the optically-induced transit to quenched domain with laser intensity initially at $87 \mathrm{~kW} / \mathrm{cm}^{2}$ then decreased suddenly to $76 \mathrm{~kW} / \mathrm{cm}^{2}$. Similarly, the additional randomness is given at which the laser intensity equals $76 \mathrm{~kW} / \mathrm{cm}^{2}$. The $T_{t \rightarrow q}(A)$ spectrum, as shown by circle symbols in Fig. 9, displays no NDD effect in this transition branch. The switching time is fixed at $1.3 \mathrm{~ns}$ when $A$ is increased from 0 to $10^{-1}$.

The findings shown in Fig. 9 can be simply realized via classical attractor dynamics. If the initial conditions were fixed, the phase trajectory from the initial state to the final state and the associated relaxation time, i.e., switching time, can be determined. It is also noted that the dynamical characteristic of the model system, i.e., the phase trajectory and the relaxation time, are not influenced by a small strength of randomness (e.g., $A$ is smaller than $10^{-3}$ ). However, $T_{q \rightarrow t}(A)$ and $T_{t \rightarrow q}(A)$ exhibit different behaviors with $10^{-3} \leq A \leq 10^{-1}$. Such a result indicates that the stability of the relaxation path in this system is quite complex. The phase trajectory from the transit mode to the quenched mode still maintains its stability and is not perturbed by the strength of white noise. Nevertheless, it's a different story for the quenched mode to the transit mode. The unstable quenched mode tends to be temporally locked in phase space due to the additional randomness, i.e., noise-enhanced stability for unstable dynamical states. Naively, stronger noise can drive the dynamical system promptly into the convergent regime of the final stable state. Therefore, noise-enhanced stability should be gradually decreased as $A$ is increased. Surprisingly, in the interval of $10^{-2} \leq A \leq 10^{-1}$ our calculations indicate that no sign of increase of $T_{q \rightarrow t}$ with respect to $A$. We believe that the NDD effect shown in $T_{q \rightarrow t}(A)$ spectrum is still an unsolved problem in stochastic processes. However, these different $T_{q \rightarrow t}(A)$ and $T_{t \rightarrow q}(A)$ spectrums possibly can be explained via local as well as global bifurcation scenarios around the transition points. According to the bifurcation analysis, catastrophic scenarios can be numerically observed both in quenched state to transit state and vice versa. A common situation for catastrophe to arise is where the system undergoes a crisis at which an attractor collides with the basin boundary separating it and another coexisting attractor (Thompson \& Stewart, 1993). If the attractor is chaotic, after the crisis, the chaotic attractor is destroyed and converted into a nonattracting chaotic saddle. A dynamical trajectory then wanders in the vicinity of the chaotic saddle for a period of time before it approaches to the other attractor. This phenomenon is known as chaotic transient (Ditto et al., 1989; Grebogi et al., 1987) which can be realized via the transition from chaotic quenched state to periodic transit state in the upper portion of Fig. 8. In order to illustrate the formation of chaotic quenched domains near the transition region with laser intensity being equal to $122 \mathrm{~kW} / \mathrm{cm}^{2}$, the bifurcation diagram of current density with local minimum 

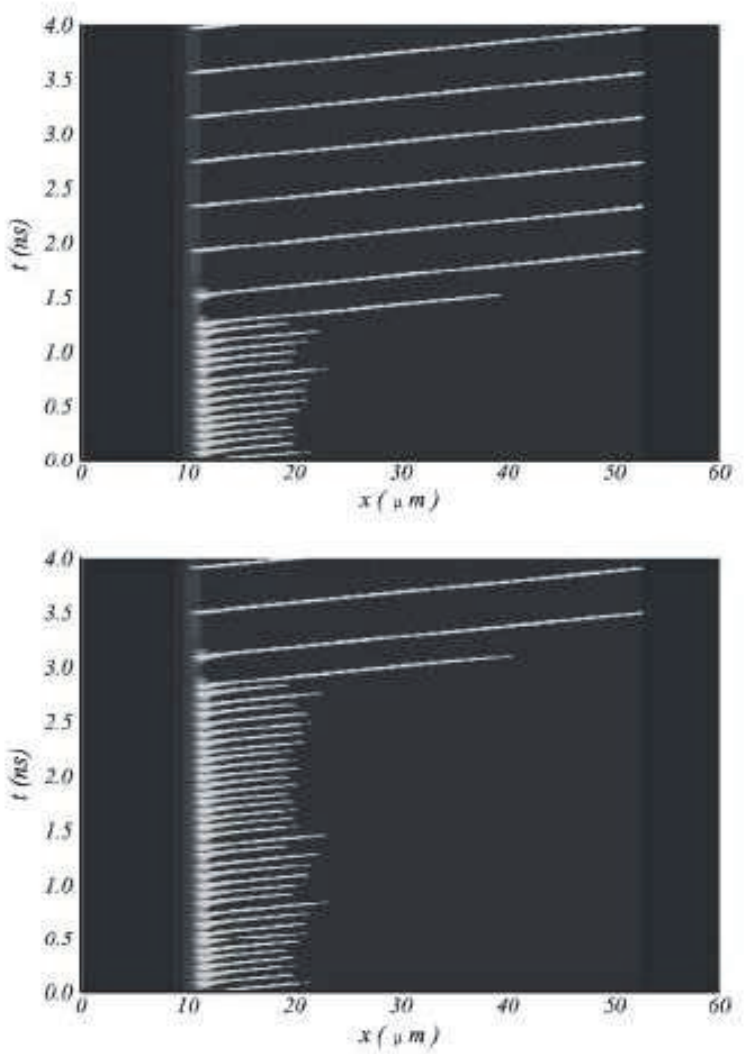

Fig. 8. The contour plots of the spatiotemporal behaviors in Fig. 7 for $A$ being equal to 0 (the upper portion) and $10^{-2}$ (the lower portion).

values $J_{b i f u r}$ versus laser intensity is illustrated in Fig. 10 which exhibits an incomplete period-doubling scenario, where $J_{b i f u r}$ is detected when the associated values is smaller than $85 \mu \mathrm{A} / \mu \mathrm{m}^{2}$. If the laser illumination with randomness is considered, the wandering time in the vicinity of the chaotic saddle will be prolonged and can be realized in the lower portion of Fig. 8. Therefore, the NDD effect in the nonlinear $T_{q \rightarrow t}(A)$ spectrum is strongly related with the dynamical structure nearby the chaotic saddle. If the attractor is periodic, after the crisis, the periodic attractor becomes unstable and quickly approaches to another coexisting attractor. The wandering time in this case should be shorter than that of the case in the vicinity of the chaotic saddle. Moreover, the dynamical structure in this case should not be perturbed by the additional weak noise. Therefore, the underlying physics for the $T_{t \rightarrow q}(A)$ spectrum with fixed and shorter values also can be interpreted as catastrophe from periodic transit state to periodic quenched state.

In summary, we introduce light-triggered spatiotemporal dynamics in layered semiconductors. Interesting phenomena shown in this Chapter include optically induced hysteresis, the photopolarization effect, exponential and non-exponential photoelectric relaxations, and long-lived transient behaviors under the consideration of optical stochasticity. 


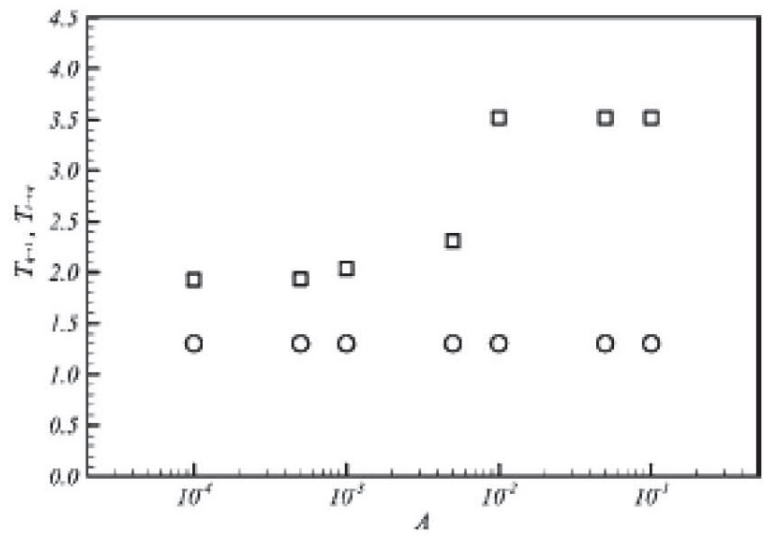

Fig. 9. Illustration of nonlinear $T_{q \rightarrow t}(A)$ and flat $T_{t \rightarrow q}(A)$ spectrums. Please note that the values of $T_{q \rightarrow t}(A)$ and $T_{t \rightarrow q}(A)$ are obtained from the ensemble average. Each switching time is averaged over 20 different realizations.

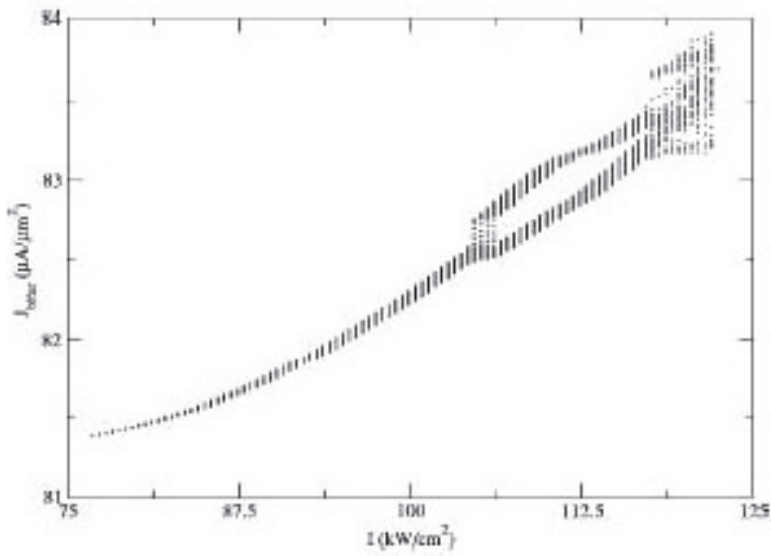

Fig. 10. The bifurcation diagram of $J_{b i f u r}$ versus $I$ represents the transition from periodic to chaotic quenched domains when laser intensity is increased.

These findings would be valueable to the development of new optoelectronics. In addition, with the assistance of nonlinear theory the operation of opto-electronic devices would be far beyond capabilities of the traditional operation.

\section{References}

Agudov, N.V. \& Malakhov, A.N. (1999). Decay of unstable equilibrium and nonequilibrium states with inverse probability current taken into account, Phys. Rev. E Vol. 60: 6333-6342.

Amann, A \& Schöll, E. (2005). Bifurcations in a system of interacting fronts, J. Stat. Phys. Vol. 119: 1069-1138. 
Bonilla, L.L. \& Grahn, H.T. (2005). Nonlinear dynamics of semiconductor superlattices, Rep. prog. Phys. Vol. 68: 577-683.

Cantalapiedra, I.R., Bergmann, M.J., Bonilla, L.L. \& Teitsworth S.W. (2001). Chaotic motion of space charge wavefronts in semiconductors under time-independent voltage bias, Phys. Rev. E Vol. 63 (No. 056216): 1-7.

Chen, C.L. (1996). Elements of Optoelectronics \& Fiber Optics, Irwin, Chicago.

Disdier, L. \& Roosen, G. (1992). Nanosecond four-wave mixing in semi-insulating GaAs, Opt. Commun. Vol. 88: 559-568.

Ditto, W.L., Rauseo, S., Cawley, R., Grebogi, C., Hsu, G.H., Kostelich, E., Ott, E., Savage, H.T., Segnan, R., Spano, M.L. \& Yorke, J.A. (1989). Experimental observation of crisis-induced intermittency and its critical exponent, Phys. Rev. Lett. Vol. 63: 923-926.

Döttling, R \& Schöll, E. (1992). Oscillatory bistability of real-space transfer in semiconductor heterostructures, Phys. Rev. B Vol. 45: 1935-1938.

Dubard, J., Smirl, A.L., Cui, A.G., Valley, G.C. \& Boggess, T.F. (1989). Beam amplification by transient energy transfer in GaAs and Si, Phys. Status Solidi (b) Vol. 150: 913-919.

Gaa, M. \& Schöll, E. (1996). Traveling carrier-density waves in n-type GaAs at low-temperature impurity breakdown, Phys. Rev. B Vol. 54: 16733-16741.

Gel'mont, B.L. \& Shur, M.S. (1970). S-type current-voltage characteristic in highly doped Gunn diodes, Sov. Phys. Solid State Vol. 12: 1304-1308.

Grebogi, C., Ott, E., Romeiras, F. \& Yorke, J.A. (1987). Critical exponents for crisis-induced intermittency, Phys. Rev. A Vol. 36: 5365-5380.

Hall, R. \& Leck, J.H. (1968). Avalanche breakdown of gallium arsenide p-n junctions, Int. J. Electron. Vol. 25: 529-537.

Horsthemke, V. \& Lefever, R. (1984). Noise Induced Transitions, Springer, Berlin.

Joshi, R.P., Kayasit, P., Islam, N., Schamiloglu, E., Fleddermann, C.B. \& Schoenberg, J. (1999). Simulation studies of persistent photoconductivity and filamentary conduction in opposed contact semi-insulating GaAs high power switches, J. Appl. Phys. Vol. 86: 3833-3843.

Oshio, K.I. \& Yahata, H. (1995). Non-periodic current oscillations in the Gunn-effect device with the impact-ionization effect, J. Phys. Soc. Jpn. Vol. 64: 1823-1836.

Schöll, E. (1987). Nonequilibrium Phase Transitions in Semiconductors, Springer, Berlin.

Shaw, M.P., Mitin, V.V., Schöll, E. \& Grubin, H.L. (1992). The Physics of Instabilities in Solid State Electron Devices, Plenum, New York.

Shiau, Y.H. \& Cheng, Y.C. (1996). Static and dynamic hysteresis in deep-impurity doped n-GaAs under d.c. bias voltage, Solid-State Electron. Vol. 39: 205-210.

Shiau, Y.H. \& Peng, Y.F. (2005). Long-lived transient behavior in an $n^{+}-n-n^{+}$semiconductor device with optical stochasticity, Phys. Rev. E Vol. 71 (No. 066216): 1-6.

Shiau, Y.H. (2006). Detection of hidden structures in a photoexcited semiconductor via principal-component analysis, Solid-State Electron. Vol. 50: 191-198.

Thompson, J.M.T. \& Stewart, H.B. (1993). Nonlinear Dynamics and Chaos, Wiley, New York.

Wacker, A. (2002). Semiconductor superlattices: A model system for nonlinear transport, Phys. Rep. Vol. 357: 1-111.

Yeh, P. (1993). Introduction to Photorefractive Nonlinear Optics, John Wiley \& Sons, New York.

Sze, S.M. (1969). Physics of Semiconductor Devices, Wiley, New York. 


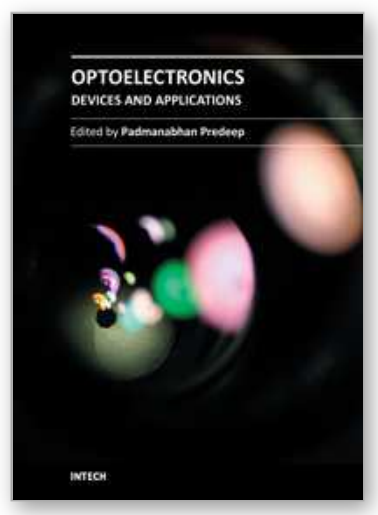

\author{
Optoelectronics - Devices and Applications \\ Edited by Prof. P. Predeep
}

ISBN 978-953-307-576-1

Hard cover, 630 pages

Publisher InTech

Published online 03, October, 2011

Published in print edition October, 2011

Optoelectronics - Devices and Applications is the second part of an edited anthology on the multifaced areas of optoelectronics by a selected group of authors including promising novices to experts in the field. Photonics and optoelectronics are making an impact multiple times as the semiconductor revolution made on the quality of our life. In telecommunication, entertainment devices, computational techniques, clean energy harvesting, medical instrumentation, materials and device characterization and scores of other areas of R\&D the science of optics and electronics get coupled by fine technology advances to make incredibly large strides. The technology of light has advanced to a stage where disciplines sans boundaries are finding it indispensable. New design concepts are fast emerging and being tested and applications developed in an unimaginable pace and speed. The wide spectrum of topics related to optoelectronics and photonics presented here is sure to make this collection of essays extremely useful to students and other stake holders in the field such as researchers and device designers.

\title{
How to reference
}

In order to correctly reference this scholarly work, feel free to copy and paste the following:

Yuo-Hsien Shiau (2011). Photopolarization Effect and Photoelectric Phenomena in Layered GaAs Semiconductors, Optoelectronics - Devices and Applications, Prof. P. Predeep (Ed.), ISBN: 978-953-307-5761, InTech, Available from: http://www.intechopen.com/books/optoelectronics-devices-andapplications/photopolarization-effect-and-photoelectric-phenomena-in-layered-gaas-semiconductors

\section{INTECH}

open science | open minds

\section{InTech Europe}

University Campus STeP Ri

Slavka Krautzeka 83/A

51000 Rijeka, Croatia

Phone: +385 (51) 770447

Fax: +385 (51) 686166

www.intechopen.com

\section{InTech China}

Unit 405, Office Block, Hotel Equatorial Shanghai

No.65, Yan An Road (West), Shanghai, 200040, China

中国上海市延安西路65号上海国际贵都大饭店办公楼405单元

Phone: +86-21-62489820

Fax: $+86-21-62489821$ 
(C) 2011 The Author(s). Licensee IntechOpen. This is an open access article distributed under the terms of the Creative Commons Attribution 3.0 License, which permits unrestricted use, distribution, and reproduction in any medium, provided the original work is properly cited. 